\title{
ONSEN shows different transposition activities in RdDM pathway mutants
}

\author{
Yui Hayashi ${ }^{1}$, Kanako Takehira ${ }^{1}$, Kosuke Nozawa ${ }^{1}$, Takamasa Suzuki ${ }^{2}$, \\ Yukari Masuta ${ }^{3}$, Atsushi Kato ${ }^{3}$ and Hidetaka Ito $^{3 *}$ \\ ${ }^{1}$ Graduate School of Life Science, Hokkaido University, Sapporo, Hokkaido 060-0810, Japan \\ ${ }^{2}$ College of Bioscience and Biotechnology, Chubu University, Kasugai, Aichi 487-8501, Japan \\ ${ }^{3}$ Faculty of Science, Hokkaido University, Sapporo, Hokkaido 060-0810, Japan
}

(Received 22 March 2020, accepted 14 April 2020; J-STAGE Advance published date: 5 September 2020)

Most transposable elements (TEs) are tightly regulated by epigenetic mechanisms such as DNA methylation. RNA-directed DNA methylation (RdDM) is a major control mechanism of TE silencing in plants. We analyzed the transposition activity of a heat-responsive retrotransposon, ONSEN, in Arabidopsis thaliana. Transgenerational transposition was observed in RdDM pathwaydeficient mutants upon heat stress. The transposition frequency was higher in the mutants of the upstream processes, but lower in the mutants of the downstream steps, of RdDM. The transposition frequency was not associated with the number of extrachromosomal ONSEN copies. Constitutive heterochromatin of interphase nuclei was dispersed upon heat stress. The degree of decondensation was higher in the RdDM mutants than in wild-type plants subjected to heat stress. We discuss the possible role of RdDM in the regulation of ONSEN transposition upon heat stress.

Key words: Arabidopsis thaliana, heat stress, ONSEN, RdDM, transposon

\section{INTRODUCTION}

The transposition of mobile elements causes mutations and may affect the expression of flanking genes, resulting in genetic variation (Kazazian, 2004; Biémont and Vieira, 2006; Pecinka et al., 2010; Casacuberta and González, 2013). In particular, retrotransposons that use RNAmediated amplification are abundant in plant genomes (Kumar and Bennetzen, 1999). LTR retrotransposons comprise long terminal repeats on either side of an inner coding region. Transposition of the LTR retrotransposon begins with RNA polymerase II (Pol II)-mediated transcription of the element from the $5^{\prime}$-LTR. The transcript functions as an mRNA and not only encodes AP, VLP, RT, $\mathrm{RH}$ and INT, but also serves as a template for reverse transcription. The translated polyprotein is assembled in a VLP encoded by the GAG domain, which the AP cleaves to release INT, RT and RH. The RT-transcribed mRNA of an active retrotransposon moves from the nucleus to the cytoplasm and is subsequently converted by reverse transcriptase to DNA in the form of extrachromosomal DNA (ecDNA). The synthesized ecDNA is

\footnotetext{
Edited by Tetsu Kinoshita

* Corresponding author. hito@mail.sci.hokudai.ac.jp

DOI: http://doi.org/10.1266/ggs.20-00019
}

bound by INT and returns to the nucleus and integrates into a new genome position. Therefore, a new identical copy of the original element is created (Hirochika, 1993; Böhmdorfer et al., 2005).

Increasing rates of transposable element (TE) transposition cause harmful genomic changes, and plants have developed epigenetic regulation machinery that can suppress TE activity. RNA-directed DNA methylation (RdDM) is the major epigenetic pathway for TE regulation in plants (Wierzbicki et al., 2008; Gao et al., 2010). RdDM was first identified in plants infected with RNA pathogens and later shown to function as a small RNA-mediated epigenetic silencer of endogenous target genes. RdDM depends on particular transcriptional machinery that involves two plant-specific RNA polymerases, RNA polymerase IV (Pol IV) and RNA polymerase V (Pol V) (Kanno et al., 2005; Onodera et al., 2005; Pontier et al., 2005). In Arabidopsis thaliana, Pol IV transcribes single-stranded RNAs at its target sites, and RNA-dependent RNA polymerase 2 (RDR2) associates with Pol IV to convert the transcripts to double-stranded RNAs. The double-stranded RNAs are processed into 24-nucleotide (nt) siRNAs by DICER-LIKE 3 (DCL3) and exported to the cytoplasm (Zhang et al., 2007; Mosher et al., 2008). Subsequently, one strand of siRNA is loaded onto an ARGONAUTE 4 (AGO4) and imported back into 
the nucleus. AGO4-loaded siRNAs guide the targeting of transcripts produced from Pol V with the assistance of members of a histone methyltransferase family, SUVH2/9, and the DDR complex, including DRD1, DMS3 and RDM1 (Zhong et al., 2012; Johnson et al., 2014). The AGO4 complex recruits the DNA methyltransferase DOMAINS REARRANGED METHYLTRANSFERASE 2 (DRM2), which catalyzes de novo DNA methylation, to the homologous genomic sites (Cao and Jacobsen, 2002; Matzke and Birchler, 2005).

Recently, non-canonical RdDM mechanisms have been reported that are associated with post-transcriptional gene silencing (PTGS). Young TEs are originally transcribed by Pol II, and RDR6 generates double-stranded precursors that are processed by DICER-LIKE 2 (DCL2) and DICER-LIKE 4 (DCL4) to generate 21- to 22-nt siRNAs. The siRNAs are loaded onto ARGONAUTE 1 (AGO1) and guide cleavage of the transposon mRNA in a typical PTGS pathway (Matzke and Mosher, 2014). Furthermore, some of the processed 21-22-nt siRNAs cooperate with ARGONAUTE 2 (AGO2), guide the transcripts produced from Pol V, and subsequently recruit DRM2, resulting in de novo DNA methylation on the activated TEs. The initial methylation catalyzed by DRM2 promotes the canonical RdDM (Nuthikattu et al., 2013). The PTGS caused by RDR6-dependent RdDM was shifted to transcriptional gene silencing (TGS), which is mediated by canonical RDR2-dependent RdDM when the 21-22-nt siRNAs overload DCL2 and DCL4 and promote processing by DCL3 to produce 24-nt siRNA (Marí-Ordóñez et al., 2013).

Most TEs are silent in normal conditions but are activated in plants as a result of environmental changes (Suoniemi et al., 1996; Wessler, 1996; Kumar and Bennetzen, 1999; Takeda et al., 1999; Beguiristain et al., 2001; Kimura et al., 2001; Salazar et al., 2007; Pecinka et al., 2010; Ito et al., 2011, 2013; Woodrow et al., 2011). The transposition of activated TEs potentially generates the genetic diversity required to enable environmental adaptations. Previously, we reported that heat stress activates an LTR retrotransposon named ONSEN in A. thaliana (Ito et al., 2011). The LTR of ONSEN contains a sequence that is recognized by the plant's heat response transcription factor, HsfA2 (Cavrak et al., 2014). HsfA2 has a conserved N-terminal DNA-binding domain that binds a heat response element in the ONSEN LTR, indicating that HsfA2 is directly involved in the transcriptional activation. ONSEN is transcribed into ecDNA that can potentially transpose. Accumulation of many ONSEN transcripts and ecDNA was observed in mutants impaired in siRNA biogenesis (Ito et al., 2011). Moreover, the transposition of ONSEN was observed in the progeny of heat-stressed mutant A. thaliana that lacked the RdDM pathway (Cavrak et al., 2014).

However, we know little about the precise mechanisms of the transpositional regulation of ONSEN. Here, we investigated the possible regulation mechanisms of ONSEN transposition upon heat stress. We found a high frequency of ONSEN transpositions in mutants of Pol IV and RDR2, but not of Pol V or DRM2, upon heat stress. Our results suggest the existence of a specific mechanism, independent of canonical RdDM, that regulates ONSEN transposition.

\section{MATERIALS AND METHODS}

Plant materials and stress conditions We used both wild-type and mutant $A$. thaliana plants in the experiments. Mutants used included nrpd1 (Herr et al., 2005), nrpe1 (Kanno et al., 2005) and the following mutants from the ABRC stock center: $r d r 2$ (CS66076), rdr6 (CS879578), dcl2 /4 (CS66078), dcl3 (CS16390), ago2 (SALK_003380), ago4 (SALK_027933C) and drm2 (CS16386). All mutants and wild-type plants used in this study were of the Columbia (Col-0) background. The plants were grown on Murashige and Skoog (MS) plates under continuous light at $21{ }^{\circ} \mathrm{C}$. For the heat stress treatment employed to analyze ONSEN expression and DNA methylation, seven-day-old seedlings were subjected to a temperature shift from $21{ }^{\circ} \mathrm{C}$ to $37^{\circ} \mathrm{C}$ for $24 \mathrm{~h}$.

Southern blot analysis Plant genomic DNA was isolated using the Nucleon PhytoPure DNA Extraction Kit (GE Healthcare) from two-week-old plants. Southern blots were performed as described previously (Miura et al., 2004). We detected hybridization signals in a highly concentrated sodium dodecyl sulfate hybridization buffer (Church and Gilbert, 1984) using a radiolabeled ONSENspecific probe (Supplementary Table S1) generated using the Amersham Megaprime DNA Labeling System (GE Healthcare).

Quantitative real-time PCR To quantify ONSEN copies, genomic DNA was extracted from seedlings using the DNeasy Plant Mini Kit (QIAGEN), according to the supplier's recommendations. Real-time PCR was performed using the Applied Biosystems 7300 Real-Time PCR System with the Power SYBR Green PCR Master Mix (Thermo Fisher Scientific). ONSEN-specific primers and internal control primers were used (Supplementary Table S1). Three biological replications were performed, and the standard deviation was determined.

Cytological analysis Young leaves were fixed in fresh ethanol/acetic acid (3:1) and stored at $4{ }^{\circ} \mathrm{C}$ overnight. The fixed leaves were rinsed in distilled water $(2 \times 5 \mathrm{~min})$ and citric buffer (10 mM sodium citrate, $\mathrm{pH} 4.8 ; 5 \mathrm{~min})$. Subsequently, the leaves were incubated in $1 \%(\mathrm{w} / \mathrm{v})$ cellulase (Yakult), pectolyase (Kyowa Chemical Products) and cytohelicase (Sigma) in citric buffer at $37{ }^{\circ} \mathrm{C}$ for $1 \mathrm{~h}$. After 
digestion, individual leaves were placed on a slide, dissected, and disintegrated using needles in $5 \mu \mathrm{l}$ of $60 \%$ acetic acid. The slide was placed on a hot plate $\left(42{ }^{\circ} \mathrm{C}\right)$ for $30 \mathrm{~s}$ and covered with a coverslip. After squashing, the slide was frozen at $-80{ }^{\circ} \mathrm{C}$ and the coverslip was removed using a razor blade. The slide was dried at room temperature, and interphase nuclei were detected by counterstaining with DAPI.

\section{RESULTS}

Transpositional activation of ONSEN in mutants deficient for RdDM RdDM plays a critical role in the regulation of ONSEN activity upon heat stress. To illuminate the function of each RdDM factor, we analyzed the transgenerational transposition of ONSEN in the progeny of heat-stressed mutants that lacked the RdDM path- way. Nine siblings from each of the three parents were analyzed in the mutant lines. We analyzed mutants related to both the canonical Pol IV-RDR2-dependent pathway (nrpd1, nrpe1, rdr2, dcl3, ago4 and drm2) and the non-canonical Pol II-RDR6-dependent pathway (rdr6, dcl2/4 and ago2). New insertions were detected only in mutants related to the Pol IV-RDR2-dependent pathway involving nrpd1, nrpe1, rdr2 and drm2 (Fig. 1A), indicating that the Pol IV-RDR2-dependent pathway regulated ONSEN transposition. To evaluate the transpositional regulation of ONSEN by the Pol IV-RDR2dependent pathway, we analyzed the transposition frequency of ONSEN in the progeny of heat-stressed plants by Southern blot. Nine siblings from each of the three parents were analyzed in the mutant lines nrpd1, nrpe1, $r d r 2$ and $d r m 2$. The transposition frequency was determined based on the total number of new bands detected in

A
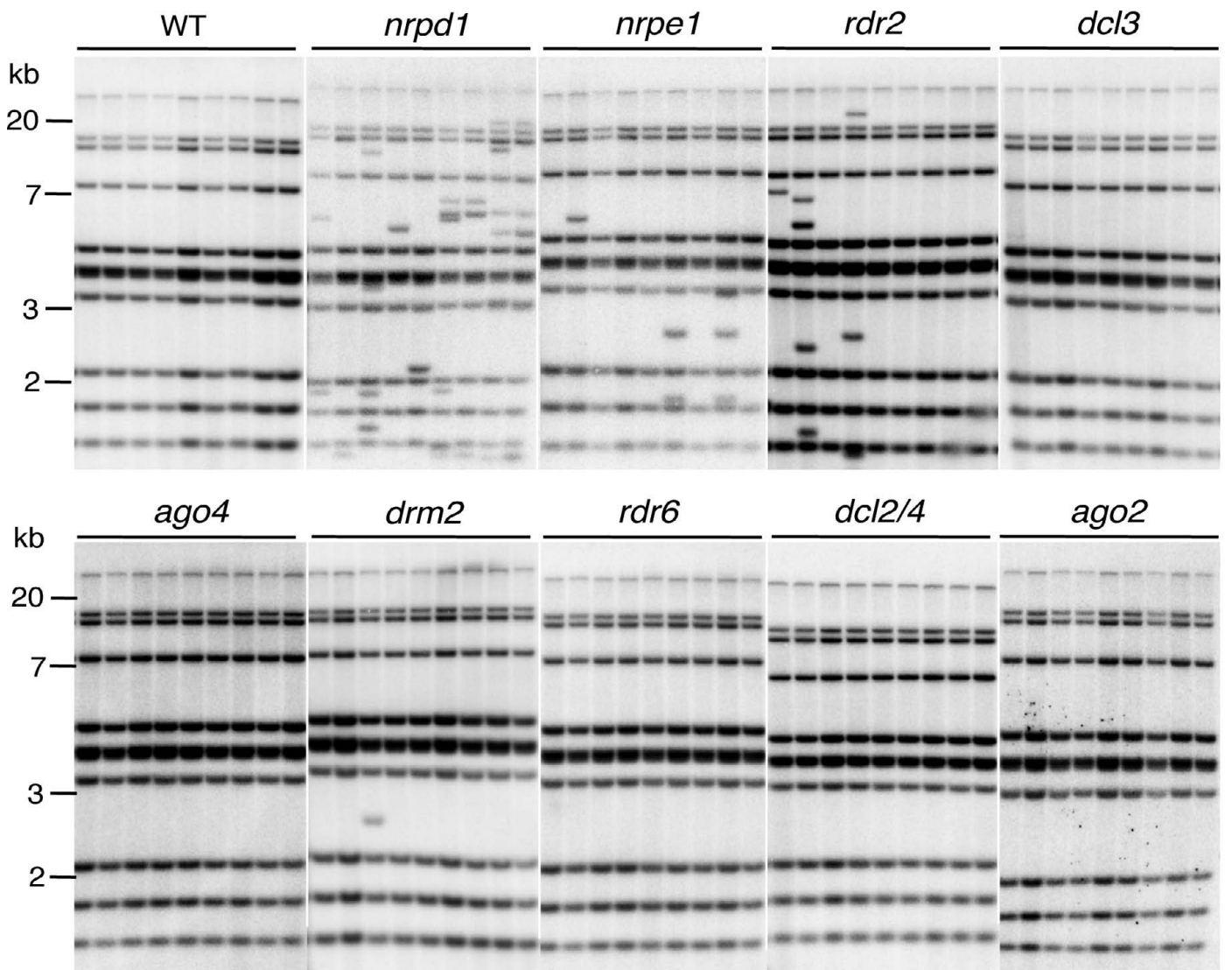

B

\begin{tabular}{c|c|c|c|c|c|c|c|c|c|c}
\hline & WT & $n r p d 1$ & $n r p e 1$ & $r d r 2$ & $d c / 3$ & $a g 04$ & $d r m 2$ & $r d r 6$ & $d c / 2 / 4$ & ago2 \\
\hline $\begin{array}{c}\text { Total } \\
\text { number }\end{array}$ & $0^{*}$ & $\begin{array}{c}23.00 \pm \\
4.24\end{array}$ & $\begin{array}{c}2.33 \pm \\
2.62^{*}\end{array}$ & $\begin{array}{c}10.00 \pm \\
8.60\end{array}$ & $0^{*}$ & $0^{*}$ & $\begin{array}{c}2.67 \pm \\
3.09^{*}\end{array}$ & $0^{*}$ & $0^{*}$ & $0^{*}$ \\
\hline
\end{tabular}

Fig. 1. (A) Southern blot analysis detects several new copies of ONSEN in the progeny of heat-stressed mutants. Each lane represents individual progeny produced from a single heat-stressed mutant. (B) The total number of new bands was determined in the progenies. Data are represented as mean \pm standard deviation $(\mathrm{n}=3)$. Asterisks mark significant differences from the number in $n r p d 1(P \leq 0.05$, ordinary one-way ANOVA). 


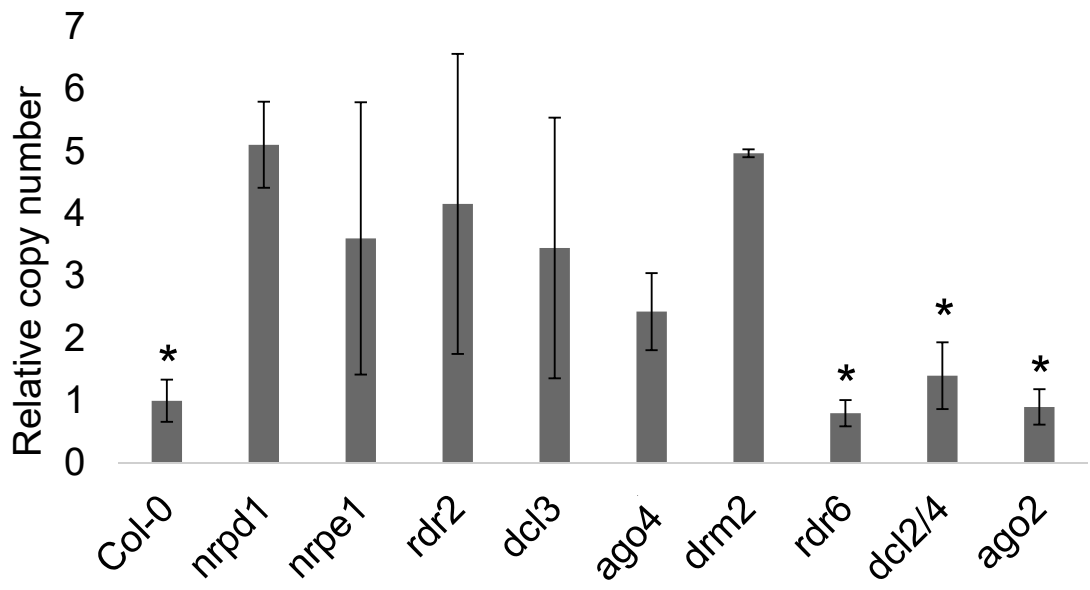

Fig. 2. Relative copy number of extrachromosomal DNA of ONSEN in heat-stressed seedlings. Error bars represent the mean $\pm \mathrm{SEM}, \mathrm{n}=3$; values are relative to heat-stressed plants (Col-0). Asterisks mark significant differences from $n r p d 1(P \leq 0.05$, Student's $t$-test).

A

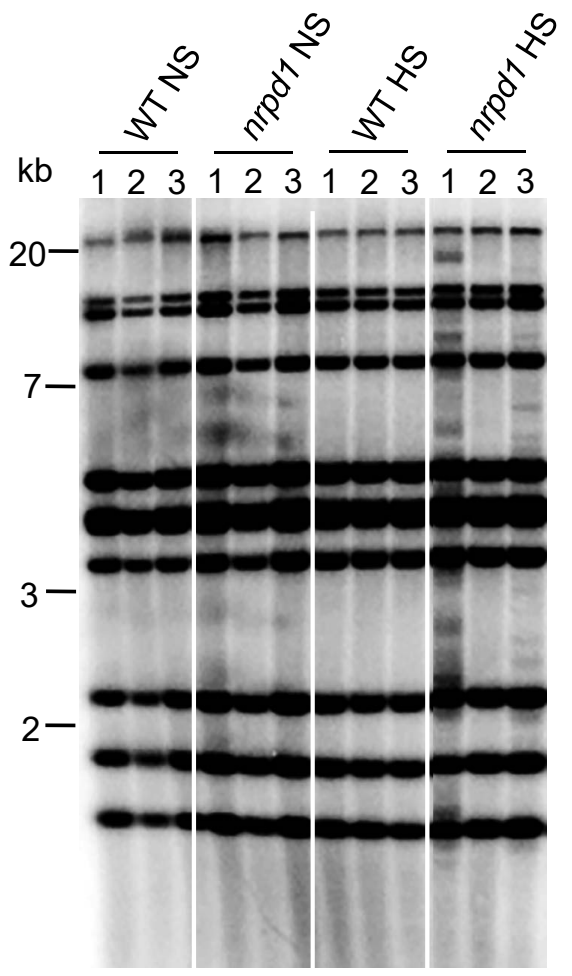

B

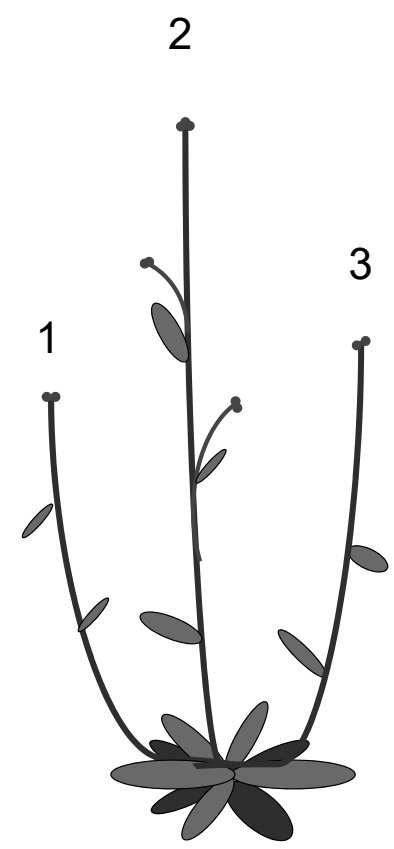

Fig. 3. ONSEN transposition observed in floral tissues upon heat stress. Southern blot analysis reveals several new copies of ONSEN in the nrpd1 scape, where all flowers were removed (A). Each lane represents an individual scape as illustrated in (B).

the siblings. The number of new bands was significantly lower in nrpe 1 and drm 2 than in nrpd1 (Fig. 1B). These results suggest that the transposition of ONSEN is primarily regulated by the Pol IV/RDR2 products and that the transposition frequency is regulated through a reproductive pathway.
The frequency of ONSEN transposition does not correlate with the copy number of ecDNA To understand the relationship between the copy number of ONSEN and transposition frequency, we determined the number of ONSEN copies in each mutant subjected to heat stress. We used real-time PCR for detecting the copy number of ONSEN that included actively released ecDNA. The results showed that the amount of ONSEN 
ecDNA accumulated was high in the mutants related to the Pol IV-RDR2-dependent pathway, including nrpd1, nrpe1, rdr2 and drm2 (Fig. 2). However, the number of ONSEN copies in nrpd1 was not significantly different from that in other mutants, indicating that the high frequency of transposition in the nrpd1 mutant was independent of the amount of accumulated ecDNA. Notably, the number of ONSEN copies in the heat-stressed mutants of the Pol II-RDR6-dependent pathway, including $r d r 6, d c l 2 / 4$ and ago2, was significantly lower than that in $\operatorname{nrpd} 1$ (Fig. 2).

High frequency of ONSEN transposition in floral tissues upon heat stress The transition from the vegetative to reproductive life stage is described as a phase change, which is associated with floral transition in $A$. thaliana (Poethig, 2003). The regulation of flowering time has been studied through the discovery of a complicated gene network and epigenetic factors (Putterill et al., 2004; Simpson, 2004; Bernier and Périlleux, 2005; Dennis and Peacock, 2007; Ojolo et al., 2018). To understand the transpositional regulation of ONSEN in the reproductive phase, we analyzed the transposition of ONSEN in the scape of heat-stressed plants. Genomic DNA was extracted from each scape, from which all flowers were removed. Southern blot analysis revealed a large number of new insertions in heat-stressed nrpd1 mutants, although no new insertions were detected in the wild type subjected to heat stress. This indicates that the new ONSEN insertions in meristematic cells propagate to reproductive tissue and are heritable by the progeny through the germline in $n r p d 1$ (Fig. 3).

Heterochromatin is decondensed in RdDM mutants upon heat stress Heat stress can give rise to altered chromatin protein complexes and changes in chromatin compaction. To test the possibility that the transposition frequency of ONSEN is affected by chromatin structure, we analyzed the chromatin condensation of wild type, nrpd1, rdr2 and drm2 using a cytological approach. We examined about 100 cells in interphase that were extracted from heat-stressed or non-stressed young leaf tissue. The number of cells that contained typical centromeric heterochromatin was evaluated, along with the heterochromatin morphology (dispersed, intermediate and condensed) captured by DAPI staining (Fig. 4). The result showed that the proportion of nuclei that contain dispersed heterochromatin upon heat stress

A

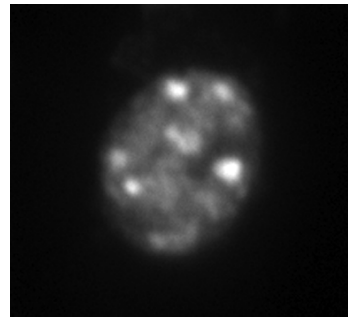

Condensed

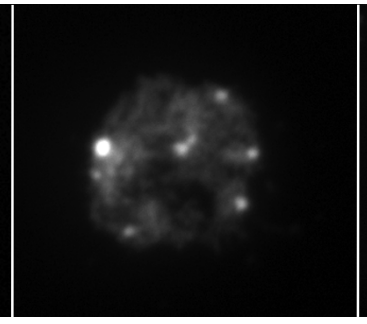

Intermediate

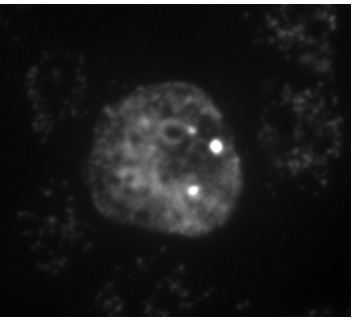

Dispersed

B

WT NS
$n r p d 1$ NS
$r d r 2$ NS
$d r m 2$ NS
WT HS
$n r p d 1$ HS
$r d r 2$ HS
$d r m 2$ HS
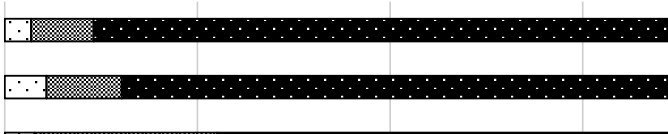

$\therefore \therefore \therefore$
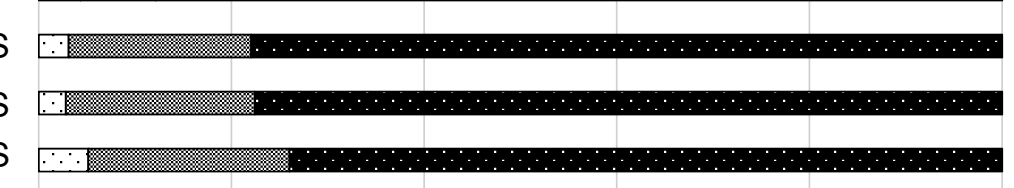

.

drm $2 \mathrm{HS}$

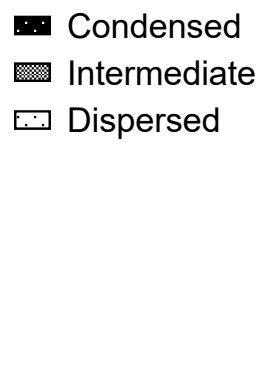

$(\%)$

Fig. 4. Heterochromatin decondensation in RdDM mutants upon heat stress. (A) The three representative phenotypes of DAPIstained interphase nuclei isolated from heat-treated young leaf tissue. The phenotype was categorized as condensed, intermediate and dispersed based on the size of the DAPI-stained heterochromatin. (B) The proportions of nuclei that contain the three categorized heterochromatins upon heat stress. About 100 interphase nuclei were analyzed in wild type and the mutants. NS: non-stressed plant, HS: heat-stressed plant. 
was higher in the canonical RdDM mutants (nrpd1, rdr2 and $d r m 2$ ) than in the wild type, although there were no differences among wild type, $n r p d 1, r d r 2$ and $d r m 2$ without heat stress (Fig. 4).

\section{DISCUSSION}

Activation of TEs is tightly regulated by epigenetic modification because disordered activation is harmful to the host genome. RdDM plays an important role in TE silencing in plants. Pol IV is essential to initiate the generation of 24-nt siRNAs, which occurs via the transcription of single-stranded RNAs at target loci. The transcribed RNAs are shorter than $50 \mathrm{nt}$, and each is processed to become a single 24-nt siRNA (Blevins et al., 2015; Zhai et al., 2015; Yang et al., 2016). The 24-nt siRNAs function via both DCL-dependent and DCLindependent pathways. In the DCL-dependent pathway, the short precursor RNAs produced by Pol IV are used as templates to synthesize the double-stranded RNAs by RDR2 and subsequently processed to form 24-nt siRNAs by DCL3. Conversely, some Pol IV-dependent short RNAs can guide DNA methylation at target loci in a DCL-independent manner (Yang et al., 2016). The multiprocessing pathway of Pol IV-dependent short RNAs indicated that there are also several pathways for transpositional regulation of TEs. In our study, the mutants of Pol IV and RDR2 showed a high frequency of ONSEN transposition in the progeny of heat-stressed plants. This result is consistent with the finding that Pol IV could not produce siRNAs without the presence of RDR2 (Blevins et al., 2015; Li et al., 2015; Zhai et al., 2015) The low transposition frequency of ONSEN in the mutants that were downstream of RdDM, including Pol V and DRM2, indicated that the transposition was regulated by multiple factors acting in the RdDM pathway. Ye et al. (2016) showed that Pol IV-dependent transcripts are capable of directing DNA methylation in the absence of 24-nt siRNAs; they noted that ONSEN transposition is partly regulated by a pathway that is independent of the canonical RdDM.

The transcriptional regulation of TEs is well studied, but transpositional regulation is poorly understood in plants. In our previous study we observed no transposition in rosette leaves of either the wild type or Pol IV mutants upon heat stress. However, a high frequency of new insertions was observed in the progeny of Pol IV mutants subjected to heat stress (Ito et al., 2011), and analysis of the insertion patterns revealed that transgenerational transposition occurred before gametogenesis (Ito et al., 2011). Here, we analyzed the somatic regulation of TEs in the tissue that changed from vegetative growth to reproductive growth. Active transposition of ONSEN was observed in the scape in Pol IV mutants but not in the wild type, suggesting that the somatic trans- position of ONSEN is regulated by Pol IV after onset of the reproductive phase.

Retrotransposons transpose through reverse transcription of their mRNA and integration of the newly created ecDNA into another location. We analyzed the effect of the amount of ecDNA on the transposition frequency of ONSEN. Transposition was unaffected by the accumulation of ecDNA, indicating that the transcription level of ONSEN is not associated with the transpositional efficiency. A previous study showed that no transgenerational transposition was observed in the suvh2 mutant, which produced higher amounts of ecDNA than the wild type (Ito et al., 2011). That observation supports the hypothesis that ONSEN transposition is not associated with the amount of ecDNA.

Transposition frequency may be affected not only by DNA methylation but also by histone modifications that could change the chromatin structure. We analyzed heterochromatin condensation in mutants of the canonical RdDM pathway. Constitutive heterochromatin was decondensed by heat stress, and the degree of dispersion was higher in the mutants than in the wild type. The decondensation of centromeric heterochromatin in the mutants is reasonable because most of the RdDM targets are centromeric transposons. The chromatin state that might be important for ONSEN insertion merits further research, although there are technical limitations for analyzing the chromatin state in a single cell in which the transposition event occurred. A recent report revealed that plants defective in Pol II activity lost DNA methylation at repeat sequences and produced additional ONSEN ecDNA upon heat stress (Thieme et al., 2017). A number of RNAs may be involved in the transpositional regulation of ONSEN, although the physical interaction between the RNAs and ecDNA is unclear.

This work was supported by NIG-JOINT (70A2019), JSPS KAKENHI (JP18K06050), and a Grant-in-Aid for scientific Research on Innovative Areas (JP15H05960).

\section{REFERENCES}

Beguiristain, T., Grandbastien, M.-A., Puigdoménech, P., and Casacuberta, J. M. (2001) Three Tnt1 subfamilies show different stress-associated patterns of expression in tobacco. Consequences for retrotransposon control and evolution in plants. Plant Physiol. 127, 212-221.

Bernier, G., and Périlleux, C. (2005) A physiological overview of the genetics of flowering time control. Plant Biotechnol. J. 3, 3-16.

Biémont, C., and Vieira, C. (2006) Junk DNA as an evolutionary force. Nature 443, 521-524.

Blevins, T., Podicheti, R., Mishra, V., Marasco, M., Wang, J., Rusch, D., Tang, H., and Pikaard, C. S. (2015) Identification of Pol IV and RDR2-dependent precursors of $24 \mathrm{nt}$ siRNAs guiding de novo DNA methylation in Arabidopsis. eLife 4, e09591.

Böhmdorfer, G., Hofacker, I. L., Garber, K., Jelenic, S., 
Nizhynska, V., Hirochika, H., Stadler, P. F., and Bachmair, A. (2005) Unorthodox mRNA start site to extend the highly structured leader of retrotransposon Tto1 mRNA increases transposition rate. RNA 11, 1181-1191.

Cao, X., and Jacobsen, S. E. (2002) Role of the arabidopsis DRM methyltransferases in de novo DNA methylation and gene silencing. Curr. Biol. 12, 1138-1144.

Casacuberta, E., and Gonzáalez, J. (2013) The impact of transposable elements in environmental adaptation. Mol. Ecol. 22, 1503-1517.

Cavrak, V. V., Lettner, N., Jamge, S., Kosarewicz, A., Bayer, L. M., and Mittelsten Scheid, O. (2014) How a retrotransposon exploits the plant's heat stress response for its activation. PLoS Genet. 10, e1004115.

Church, G. M., and Gilbert, W. (1984) Genomic sequencing. Proc. Natl. Acad. Sci. USA 81, 1991-1995.

Dennis, E. S., and Peacock, W. J. (2007) Epigenetic regulation of flowering. Curr. Opin. Plant Biol. 10, 520-527.

Gao, Z., Liu, H.-L., Daxinger, L., Pontes, O., He, X., Qian, W., Lin, H., Xie, M., Lorkovic, Z. J., Zhang, S., et al. (2010) An RNA polymerase II- and AGO4-associated protein acts in RNAdirected DNA methylation. Nature 465, 106-109.

Herr, A. J., Jensen, M. B., Dalmay, T., and Baulcombe, D. C. (2005) RNA polymerase IV directs silencing of endogenous DNA. Science 308, 118-120.

Hirochika, H. (1993) Activation of tobacco retrotransposons during tissue culture. EMBO J. 12, 2521-2528.

Ito, H., Gaubert, H., Bucher, E., Mirouze, M., Vaillant, I., and Paszkowski, J. (2011) An siRNA pathway prevents transgenerational retrotransposition in plants subjected to stress. Nature 472, 115-119.

Ito, H., Yoshida, T., Tsukahara, S., and Kawabe, A. (2013) Evolution of the ONSEN retrotransposon family activated upon heat stress in Brassicaceae. Gene 518, 256-261.

Johnson, L. M., Du, J., Hale, C. J., Bischof, S., Feng, S., Chodavarapu, R. K., Zhong, X., Marson, G., Pellegrini, M., Segal, D. J., et al. (2014) SRA- and SET-domain-containing proteins link RNA polymerase V occupancy to DNA methylation. Nature 507, 124-128.

Kanno, T., Huettel, B., Mette, M. F., Aufsatz, W., Jaligot, E., Daxinger, L., Kreil, D. P., Matzke, M., and Matzke, A. J. M. (2005) Atypical RNA polymerase subunits required for RNA-directed DNA methylation. Nat. Genet. 37, 761-765.

Kazazian, H. H., Jr. (2004) Mobile elements: drivers of genome evolution. Science 303, 1626-1632.

Kimura, Y., Tosa, Y., Shimada, S., Sogo, R., Kusaba, M., Sunaga, T., Betsuyaku, S., Eto, Y., Nakayashiki, H., and Mayama, S. (2001) OARE-1, a Ty1-copia retrotransposon in oat activated by abiotic and biotic stresses. Plant Cell Physiol. 42, $1345-1354$

Kumar, A., and Bennetzen, J. L. (1999) Plant retrotransposons. Annu. Rev. Genet. 33, 479-532.

Li, S., Vandivier, L. E., Tu, B., Gao, L., Won, S. Y., Li, S., Zheng, B., Gregory, B. D., and Chen, X. (2015) Detection of Pol IV/RDR2-dependent transcripts at the genomic scale in Arabidopsis reveals features and regulation of siRNA biogenesis. Genome Res. 25, 235-245.

Marí-Ordóñez, A., Marchais, A., Etcheverry, M., Martin, A., Colot, V., and Voinnet, O. (2013) Reconstructing de novo silencing of an active plant retrotransposon. Nat. Genet. 45, 1029-1039.

Matzke, M. A., and Birchler, J. A. (2005) RNAi-mediated pathways in the nucleus. Nat. Rev. Genet. 6, 24-35.

Matzke, M. A., and Mosher, R. A. (2014) RNA-directed DNA methylation: an epigenetic pathway of increasing complex- ity. Nat. Rev. Genet. 15, 394-408.

Miura, A., Kato, M., Watanabe, K., Kawabe, A., Kotani, H., and Kakutani, T. (2004) Genomic localization of endogenous mobile CACTA family transposons in natural variants of Arabidopsis thaliana. Mol. Genet. Genomics 270, 524-532.

Mosher, R. A., Schwach, F., Studholme, D., and Baulcombe, D. C. (2008) PolIVb influences RNA-directed DNA methylation independently of its role in siRNA biogenesis. Proc. Natl. Acad. Sci. USA 105, 3145-3150.

Nuthikattu, S., McCue, A. D., Panda, K., Fultz, D., DeFraia, C., Thomas, E. N., and Slotkin, R. K. (2013) The initiation of epigenetic silencing of active transposable elements is triggered by RDR6 and 21-22 nucleotide small interfering RNAs. Plant Physiol. 162, 116-131.

Ojolo, S. P., Cao, S., Priyadarshani, S. V. G. N., Li, W., Yan, M., Aslam, M., Zhao, H., and Qin, Y. (2018) Regulation of plant growth and development: a review from a chromatin remodeling perspective. Front. Plant Sci. 9, 1232.

Onodera, Y., Haag, J. R., Ream, T., Costa Nunes, P., Pontes, O., and Pikaard, C. S. (2005) Plant nuclear RNA polymerase IV mediates siRNA and DNA methylation-dependent heterochromatin formation. Cell 120, 613-622.

Pecinka, A., Dinh, H. Q., Baubec, T., Rosa, M., Lettner, N., and Mittelsten Scheid, O. (2010) Epigenetic regulation of repetitive elements is attenuated by prolonged heat stress in Arabidopsis. Plant Cell 22, 3118-3129.

Poethig, R. S. (2003) Phase change and the regulation of developmental timing in plants. Science 301, 334-336.

Pontier, D., Yahubyan, G., Vega, D., Bulski, A., Saez-Vasquez, J., Hakimi, M.-A., Lerbs-Mache, S., Colot, V., and Lagrange, T. (2005) Reinforcement of silencing at transposons and highly repeated sequences requires the concerted action of two distinct RNA polymerases IV in Arabidopsis. Genes Dev. 19, 2030-2040.

Putterill, J., Laurie, R., and Macknight, R. (2004) It's time to flower: the genetic control of flowering time. BioEssays $\mathbf{2 6}$, 363-373.

Salazar, M., González, E., Casaretto, J. A., Casacuberta, J. M., and Ruiz-Lara, S. (2007) The promoter of the TLC1.1 retrotransposon from Solanum chilense is activated by multiple stress-related signaling molecules. Plant Cell Rep. 26, 1861-1868.

Simpson, G. G. (2004) The autonomous pathway: epigenetic and post-transcriptional gene regulation in the control of Arabidopsis flowering time. Curr. Opin. Plant Biol. 7, 570-574.

Suoniemi, A., Anamthawat-Jónsson, K., Arna, T., and Schulman, A. H. (1996) Retrotransposon BARE-1 is a major, dispersed component of the barley (Hordeum vulgare L.) genome. Plant Mol. Biol. 30, 1321-1329.

Takeda, S., Sugimoto, K., Otsuki, H., and Hirochika, H. (1999) A 13-bp cis-regulatory element in the LTR promoter of the tobacco retrotransposon Tto1 is involved in responsiveness to tissue culture, wounding, methyl jasmonate and fungal elicitors. Plant J. 18, 383-393.

Thieme, M., Lanciano, S., Balzergue, S., Daccord, N., Mirouze, M., and Bucher, E. (2017) Inhibition of RNA polymerase II allows controlled mobilisation of retrotransposons for plant breeding. Genome Biol. 18, 134

Wessler, S. R. (1996) Turned on by stress. Plant retrotransposons. Curr. Biol. 6, 959-961.

Wierzbicki, A. T., Haag, J. R., and Pikaard, C. S. (2008) Noncoding transcription by RNA polymerase $\mathrm{Pol} \mathrm{IVb} / \mathrm{Pol} \mathrm{V}$ mediates transcriptional silencing of overlapping and adjacent genes. Cell 135, 635-648. 
Woodrow, P., Pontecorvo, G., Ciarmiello, L. F., Fuggi, A., and Carillo, P. (2011) Ttd1a promoter is involved in DNAprotein binding by salt and light stresses. Mol. Biol. Rep. 38, 3787-3794.

Yang, D. L., Zhang, G., Tang, K., Li, J., Yang, L., Huang, H., Zhang, H., and Zhu, J. K. (2016) Dicer-independent RNAdirected DNA methylation in Arabidopsis. Cell Res. 26, $66-82$.

Ye, R., Chen, Z., Lian, B., Rowley, M. J., Xia, N., Chai, J., Li, Y., He, X.-J., Wierzbicki, A. T., and Qi, Y. (2016) A dicerindependent route for biogenesis of siRNAsthat direct DNA methylation in Arabidopsis. Mol. Cell 61, 222-235.
Zhai, J., Bischof, S., Wang, H., Feng, S., Lee, T.-f., Teng, C., Chen, X., Park, S. Y., Liu, L., Gallego-Bartolome, J., et al. (2015) A one precursor one siRNA model for Pol IV-dependent siRNA biogenesis. Cell 163, 445-455.

Zhang, X., Henderson, I. R., Lu, C., Green, P. J., and Jacobsen, S. E. (2007) Role of RNA polymerase IV in plant small RNA metabolism. Proc. Natl. Acad. Sci. USA 104, 4536-4541.

Zhong, X., Hale, C. J., Law, J. A., Johnson, L. M., Feng, S., Tu, A., and Jacobsen, S. E. (2012) DDR complex facilitates global association of RNA polymerase $\mathrm{V}$ to promoters and evolutionarily young transposons. Nat. Struct. Mol. Biol. 19, 870-875. 